\title{
Chemoselective Surface Immobilization of Proteins through a Cleavable Peptide
}

\author{
Monica Dettin, ${ }^{*, \S}$ Natali Muncan, ${ }^{\S}$ Antonella Bugatti, ${ }^{\ddagger}$ Francesca Grezzo, ${ }^{\S}$ Roberta Danesin, ${ }^{\S}$ and \\ Marco Rusnati ${ }^{\ddagger}$ \\ ${ }^{\S}$ Department of Chemical Process Engineering, University of Padova, Padova, Italy \\ ${ }^{\ddagger}$ Department of Biomedical Sciences and Biotechnology, University of Brescia, Brescia, Italy \\ S Supporting Information
}

ABSTRACT: Surface immobilization of biomolecules is a fundamental step in several experimental techniques such as surface plasmon resonance analysis and microarrays. Oxime ligation allows reaching chemoselective protein immobilization with the retention of native-like conformation by proteins. Beside the need for chemoselective ligation of molecules to surface/particle, equally important is the controlled release of the immobilized molecules, even after a specific binding event. For this purpose, we have designed and assessed in an SPR experiment a peptide linker able to (i) anchor a given protein

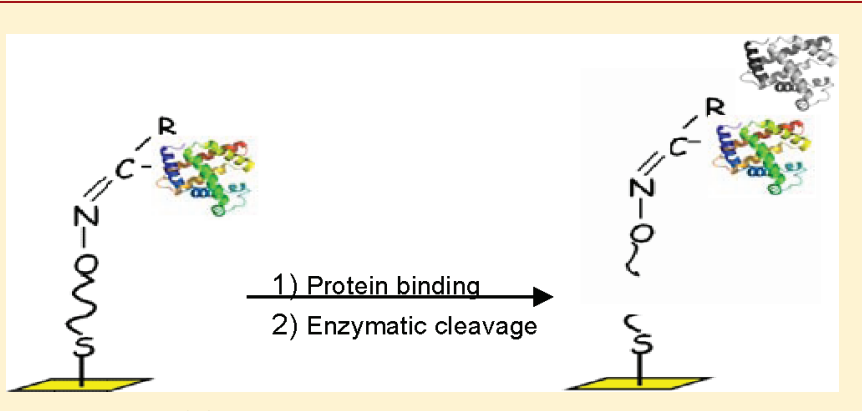
(enzymes, receptors, or antibodies) to a surface in a precise orientation and (ii) release the immobilized protein after selective enzymatic cleavage. These results open up the possibility to anchor to a surface a protein probe leaving bioactive sites free for interaction with substrates, ligands, antigens, or drugs and successively remove the probe-ligand complex by enzymatic cleavage. This peptide linker can be considered both an improvement of SPR analysis for macromolecular interaction and a novel strategy for drug delivery and biomaterial developments.

Curface immobilization of biomolecules is a key step in im$\checkmark$ portant experimental techniques including surface plasmon resonance (SPR) analysis ${ }^{1}$ and microarrays ${ }^{2}$ that are by now fundamental in research areas spanning from basic sciences to drug development. Surface functionalization with proteins is far more complex than with DNA, mainly due to the high number of different functional groups and to the structural complexity of folded proteins. Also, the need for an appropriate orientation of the immobilized protein on the surface is crucial for receptors, antibodies, and enzymes. ${ }^{3}$ For these immobilizations, leaving the binding site or the catalytic pocket free can be satisfied only by chemoselective methods. An ideal chemoselective protein immobilization might exploit a selected functional group of the protein and may use experimental conditions that retain protein folding and its biological functions. Relevant to this point, classical protein immobilization via amine or thiol groups usually results in random conjugation without a defined protein orientation. On the other hand, among the various site-specific immobilization methods available, the bioorthogonal ligation reactions, like Staudinger ligation or Diels-Alder reaction, need the introduction of a peptide tag or a non-natural amino acid that may greatly affect protein folding and activity. Lempens et al. recently proposed oxime ligation as efficient protein immobilization strategy. ${ }^{4}$ This method requests the site-specific introduction of a ketone in the $\mathrm{N}$-terminal site of the protein by oxidation with pyridoxal- $5^{\prime}$-phosphate (PLP); ${ }^{5,6}$ the ketone is then used to link, through an oxime bond, an oxy-amine group on the surface.
The conversion of the N-terminal group in ketone or aldehyde can be applied to many proteins without recombinant modification with the exclusion of those containing $\mathrm{Q}, \mathrm{W}, \mathrm{H}, \mathrm{P}$, or $\mathrm{K}$ as $\mathrm{N}$-terminal residue or proteins whose $\mathrm{N}$-terminus is blocked by post-translational modifications. Oxime ligation provides a fast and selective method for peptide and protein immobilization and its application has been demonstrated to increase the sensitivity of SPR analysis. ${ }^{7}$

Beside the need for chemoselective immobilization of molecules to surface/particle, equally important is the controlled release of the immobilized molecules (i.e., drugs) to target tissues/ cells. This latter field of research has direct implications in the treatment of several important human diseases including cancer. ${ }^{8,9}$

On the basis of the above-mentioned considerations, we have attempted the design of a bifunctional peptide linker containing the matrix metallo proteinase (MMP)-2-cleavage site of CCL12 that could be selectively bound to a protein-ketone through its oxy-amine $\mathrm{N}$-terminal group and anchored to a surface (i.e., a gold chip) through the thiol of its C-terminal Cys (Figure 1). The goal of this kind of strategy is twofold, probing both the possibility to chemoseletively immobilize a protein on a surface in a defined orientation and the possibility to collect the probe-ligand complex for further analyses and studies. Closely related to this

\section{Received: $\quad$ May 13, 2011}

Revised: July 25, 2011 


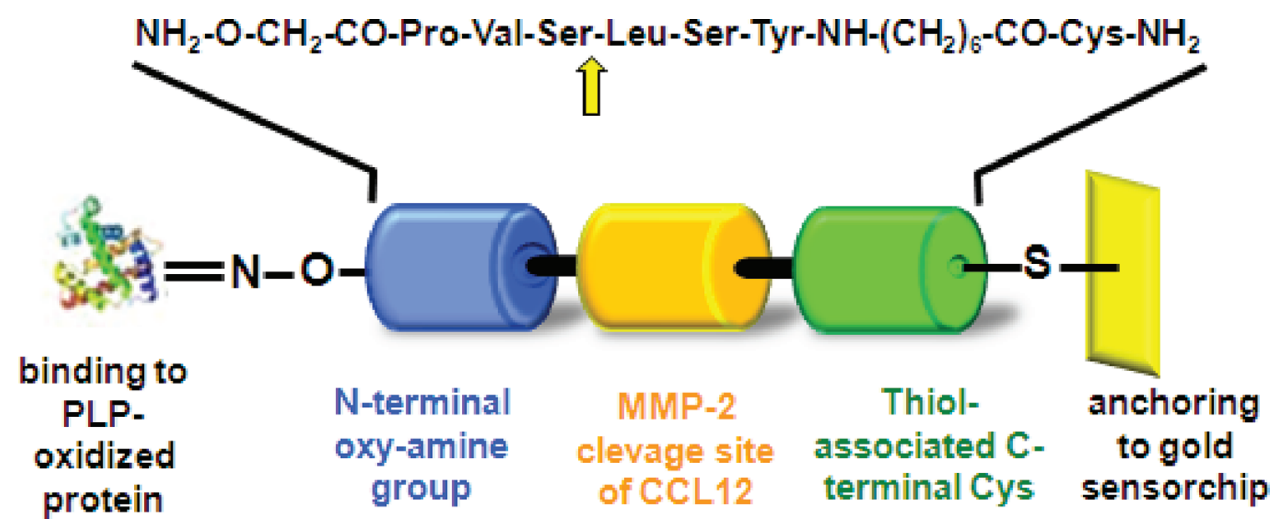

Figure 1. Schematic representation of the bifunctional peptide linker containing the MMP-2-cleavage site of CCL12.

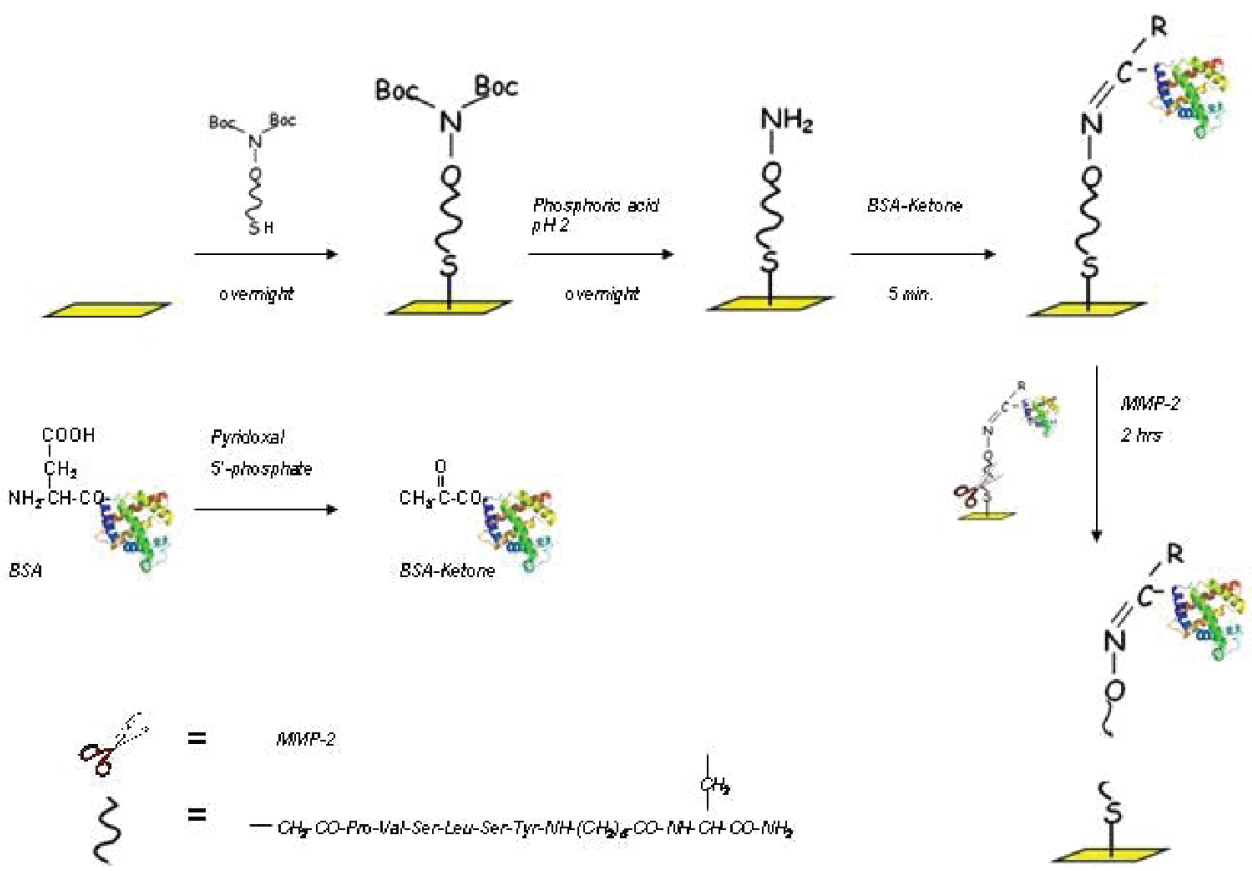

Figure 2. Schematic representation of the strategy for site-specific immobilization of proteins via a cleavable peptide linker here adopted. Boc $=$ tertbutyloxycarbonyl.

latter point, the study performed here opens new possibilities in the field of controlled release of drugs from artificial surfaces for biomedical uses.

Figure 2 reports a schematic representation of the strategy adopted here. In detail, a premature and uncontrolled reaction of the highly reactive oxy-amino group of the peptide linker was avoided by protecting it with bis-tert-butyloxycarbonyl (Bis-Boc). The solid-phase synthesis of Bis-Boc-protected peptide linker was possible using super acid labile Mmt for Cys, 2-Cl-Trt for Tyr, and Trt for Ser instead of acid labile side chain protections and a super acid labile support (Sieber Amide resin). As this group is highly reactive with aldehyde, ketones, and nitriles, acetonitrile was avoided as eluent during HPLC purification.The Bis-Boc-protected peptide linker was then immobilized to a gold-coated sensorchip for SPR analysis via thiol group of its C-terminal Cys residue. Bovine serum albumin (BSA, here used as model protein) was PLP oxidized, obtaining the BSA-Ketone, and the sensorchip-immobilized peptide linker deprotected. Then, the chemoselective fusion to the oxy-amine $\mathrm{N}$-terminal group of the immobilized peptide linker to BSA-Ketone was performed directly in the fluidic cell of the BIAcore apparatus (for technical details, see Experimental Section in the Supporting Information). The resulting sensorchip was then used to evaluate the possibility to remove BSA from the sensorchip by injecting MMP-2.

The sensorgram of a representative experiment (out of four other that gave similar results) is reported in Figure 3. The immobilization of the Bis-Boc-protected peptide linker produces a RU increase of 1513 units (approximately equal to $129 \mathrm{pmol} / \mathrm{cm}^{2}$ ). The further functionalization of the surface with BSA-Ketone through oxime formation shows a further RU increase equal to 1165 units, that approximately corresponds to $1.75 \mathrm{pmol} / \mathrm{cm}^{2}$ (thus with a reaction yield equal to $1 \%$ with respect to the anchored peptide surface density).

Addition of MMP-2 to the functionalized chip surface produces a transient, slight increase of RU that is possibly due to the association of the enzyme to the substrate. Relevant to this point, 


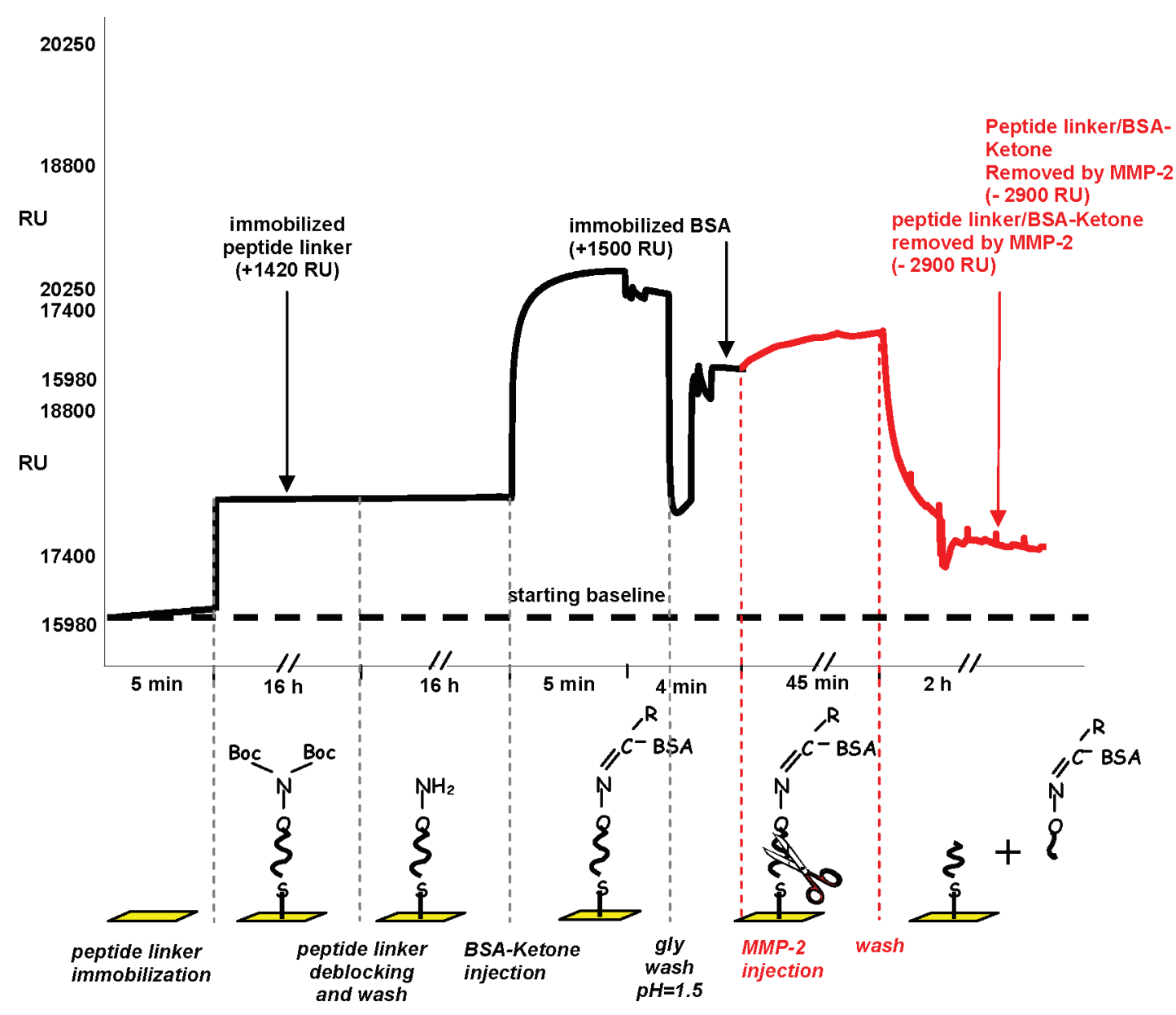

Figure 3. BIAcore responses during the procedure of chemoselective immobilization of BSA to a gold sensorchip followed by the enzymatic cleavage of the peptide linker. Boc $=$ tert-butyloxycarbonyl.

the interaction of MMP-2 to CCL12 has been demonstrated to be very strong. ${ }^{10}$ The slight increase of RU is, however, followed by a rapid decrement that reaches the level of RUs present on the sensorchip before BSA-Ketone injection.

To demonstrate the specificity of the protein association/ cleavage observed, we injected BSA-Ketone on a gold sensorchip devoid of the peptide linker. In these experimental conditions, we observed an increase of RU equal to about $800 \mathrm{RU}$ (due to unspecific adsorption of the protein to the gold surface ${ }^{11}$ ), but the subsequent treatment with MMP-2 did not produce any modification, in agreement with the lack of cleavable linker (data not shown). Relevant to this point, the observation that MMP2 completely removes peptide linker-associated BSA-Ketone suggests that, in our experimental conditions, no unspecific absorption of BSA-Ketone to the gold surface occurs, possibly due to the fact that the previous immobilization of the peptide linker has saturated the unspecific binding sites available on the gold surface. Finally, the reliability of the strategy proposed here is proven by the reproducibility of the results obtained in four repeated independent experiments (Figure 4).

In this paper, we have successfully designed and produced a bifunctional cleavable peptide that is able to efficiently link a protein to a gold surface through chemoselective immobilization and that effectively allows the controlled release of the protein by administering the appropriate enzyme. These results open up the possibility to immobilize to a surface enzymes, receptors, or antibodies with a precise orientation, leaving bioactive sites free for interaction with substrates, ligands, antigens, or drugs. This may impact the field of SPR analysis that, in the past years, has emerged as a powerful asset in the study of biomolecular interactions and drug development. ${ }^{12}$ Actually, the strategy adopted here requires the exploitation of the $\mathrm{N}$-terminus of the protein, hampering the immobilization of receptors in the proper orientation that would allow their interaction with extracellular ligands. Relevant to this point, alternative strategies have been individuated to overcome this limit: Carrico et al. have developed methods for site-specific introduction of aldehyde groups into recombinant proteins using the 6-amino-acid consensus sequence recognized by the formylglycine generating enzyme. ${ }^{13}$ On the other hand, the N-terminal immobilization of the cytoplasmic tail of a receptors may be functional to the study of receptor interaction with intracellular second messengers or intracellular binders/inhibitors. Relevant to this point, tyrosine-kinase (TK) receptors play pivotal roles in important human diseases such as tumors $^{14}$ and the development of small, cell-permeable drugs that bind the intracellular portion of TK receptors (hampering their activation and/or the docking of second messengers) represents a promising therapeutical strategy. ${ }^{15}$ In this field, the availability of a fast, reliable method for N-terminal chemoselective immobilization of TK receptors in SPR or other solid-phase assays would represent a formidable opportunity, especially for high-throughput screening of putative receptor binders/inhibitors. 


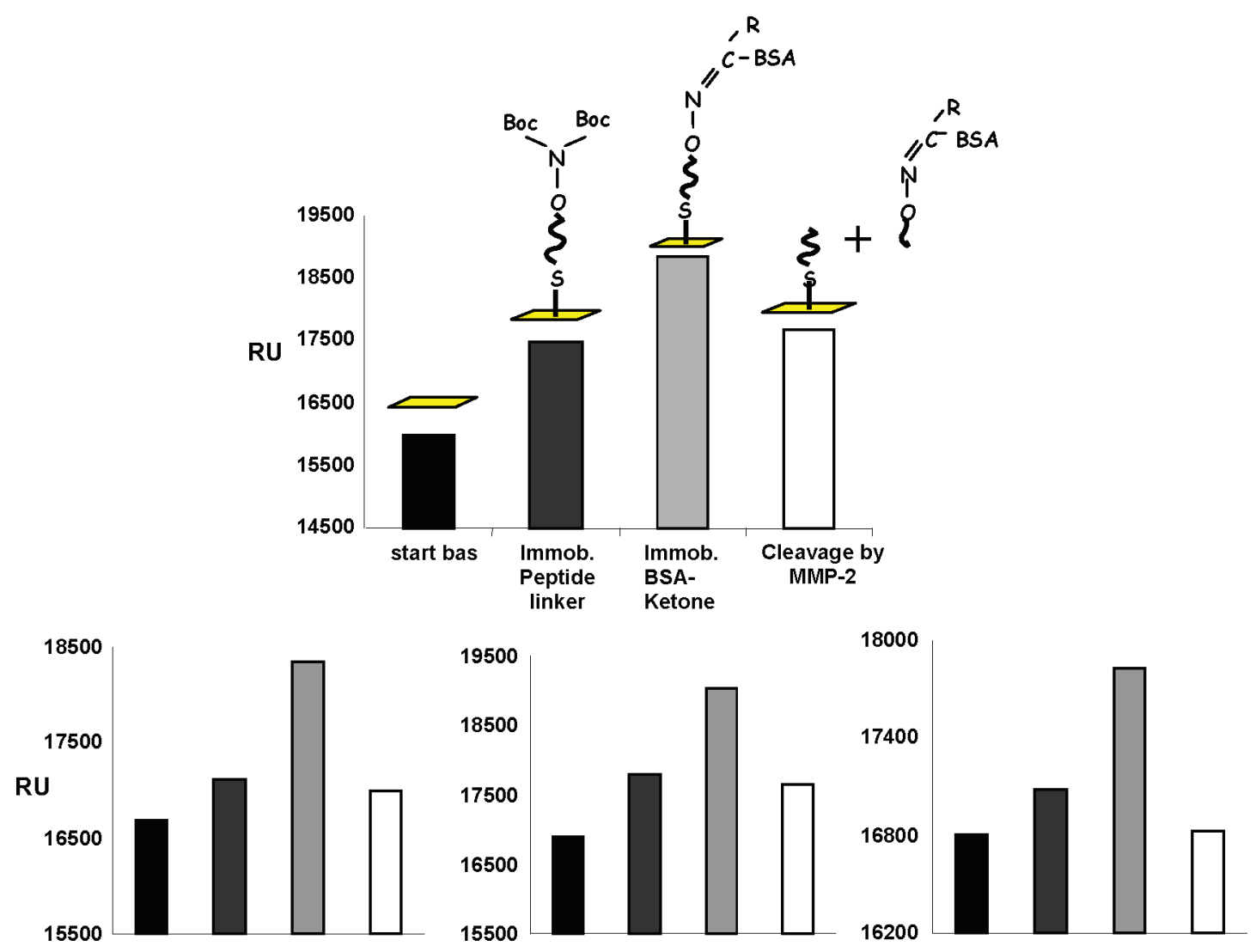

Figure 4. Immobilization levels for each of the four experiments performed. Boc = tert-butyloxycarbonyl.

Another promising evolution path that originates from the results presented here is the specific cleavage of a peptide linker with the consequent controlled release of the associated compound. Relevant to this point, the delivery and controlled release of drugs from artificial particles is an expanding field of research with implications in the treatment of several important human diseases including cancer. ${ }^{8,9}$ Interestingly, our peptide linker is sensitive to MMP2. MMP2 is a zinc-dependent endopeptidase overexpressed in advanced stages of cancer where it plays a crucial role in tumor progression, angiogenesis, and metastasis. ${ }^{16}$ It is thus possible to hypothesize that an anticancer drug immobilized to a particle via our peptide linker may be spontaneously and specifically released in MMP2-containing lesions. Also, our MMP2-labile peptide linker may behave in a "bioresponsive way", tuning drug release on the concentration of the metalloprotease that, in turn, is proportional to the extension/severity of the cancer lesion. ${ }^{16}$

Besides the approach reported here, a large number of advanced synthetic techniques have recently become available to achieve protein immobilization and modification with positional control. For example, the direct incorporation of noncanonical amino acids into proteins can be carried out via the subversion of the biosynthetic machinery: ${ }^{17}$ the subsequent coupling of synthetic molecules to artificial amino acid side chains can yield novel bioconjugates. In an alternative approach, that mimics post-translational modifications, synthetic transformations can convert specific amino acid residues on expressed natural protein into other native or modified native groups. ${ }^{18}$ When there is the necessity of protein labeling in a highly complex environment, new enzymatic strategies, able to append chemical functional groups to specific sequences, can be used. ${ }^{19}$ In addition, antibodies represent another important class of bioconjugation targets: novel methods for the selective modification of antibodies are presented in the review of Meares et al. ${ }^{20}$

In conclusion, in this paper we have designed and produced a peptide linker able to (i) anchor a given protein to a surface in a precise orientation and (ii) release the immobilized protein after selective enzymatic cleavage. As it is, our peptide linker can be considered both an improvement of SPR analysis for macromolecular interaction and a novel strategy for drug delivery and biomaterial developments.

\section{ASSOCIATED CONTENT}

S Supporting Information. Entire experimental section, including peptide synthesis, surface functionalization and BIAcore experiments. This material is available free of charge via the Internet at http://pubs.acs.org.

\section{AUTHOR INFORMATION}

\section{Corresponding Author}

*Prof. Monica Dettin, Department of Chemical Process Engineering, University of Padova, Via Marzolo, 935131 Padova, Italy. Tel. +39-049-8275553. Fax +39-049-8275555. E-mail: monica. dettin@unipd.it.

\section{ACKNOWLEDGMENT}

This work was supported by grants from MIUR, ISS (AIDS Project), and CARIPLO (grant 2008-2198) to M.R. The authors gratefully acknowledge support from the University of Padova 
through the PLATFORMS strategic project "PLAsmonic nanoTextured materials and architectures FOR enhanced Molecular Sensing" -prot.STPD089KSC.

\section{REFERENCES}

(1) Jason-Moller, L., Murphy, M., and Bruno, J. (2006) Overview of Biacore systems and their applications. In Current Protocols in Protein Science, Chapter 19, Unit 1913.

(2) Spisak, S., and Guttman, A. (2009) Biomedical applications of protein microarrays. Curr. Med. Chem. 16, 2806-2815.

(3) Kausaite-Minkstimiene, A., Ramanaviciene, A., Kirlyte, J., and Ramanavicius, A. (2010) Comparative study of random and oriented antibody immobilization techniques on the binding capacity of immunosensor. Anal. Chem. 82, 6401-6408.

(4) Lempens, E. H. M., Helms, B. A., Merkx, M., and Meijer, E. W. (2009) Efficient and chemoselective surface immobilization of proteins by using aniline-catalyzed oxime chemistry. ChemBioChem 10, 658-662.

(5) Gilmore, J. M., Scheck, R. A., Esser-Kahn, A. P., Joshi, N. S., and Francis, M. B. (2006) N- terminal protein modification through a biomimetic transamination reaction. Angew. Chem. Int. 45, 5307-5311.

(6) Scheck, R. A., Dedeo, M. D., Iavarone, A. T., and Francis, M. B. (2008) Optimization of a biomimetic transamination reaction. J. Am. Chem. Soc. 130, 11762-11770.

(7) Segers, V. F. M., Tokunou, T., Higgins, L. J., MacGillivray, C., Gannon, J., and Lee, R. T. (2007) Local delivery of protease-resistant stromal cell derived factor- 1 for stem cell recruitment after myocardial infarction. Circulation 116, 1683-1692.

(8) Ogi, H., Fukunishi, Y., Nagai, H., Okamoto, K., Hirao, M., and Nishiyama, M. (2009) Nonspecific-adsorption behavior of polyethylenglycol and bovine serum albumin studied by $55-\mathrm{MHz}$ wireless-electrodeless quartz crystal microbalance. Biosens. Bioelectron. 24, 3148-3152.

(9) Rich, R. L., and Myszka, D. G. (2009) Grading the commercial optical biosensor literature-Class of 2008: 'The Mighty Binders'. J. Mol. Recognit. 23, 1-64.

(10) McQuibban, G. A., Butler, G. S., Gong, J. H., Bendall, L., Power, C., Clark-Lewis, I., and Overall, C. M. (2001) Matrix metalloproteinase activity inactivates the CXC chemokine stromal cell-derived factor- 1 . J. Biol. Chem. 276, 43503-43508.

(11) Lemmon, M. A., and Schlessinger, J. (2010) Cell signaling by receptor tyrosine kinases. Cell 141, 1117-1134.

(12) Sharma, P. S., Sharma, S., and Tyagi, T. (2009) Receptor tyrosine kinase inhibitors as potent weapons in war against cancers. Curr. Pharm. Des. 15, 758-776.

(13) Carrico, I. S., Carlson, B. L., and Bertozzi, C. R. (2007) Introducing genetically encoded aldehydes into proteins. Nat. Chem. Biol. 3, 321-322.

(14) Wang, M., and Thanou, M. (2010) Targeting nanoparticles to cancer. Pharmacol. Res. 62, 90-99.

(15) Gullotti, E., and Yeo, Y. (2009) Extracellularly activated nanocarriers: a new paradigm of tumor targeted drug delivery. Mol. Pharm. 6, 1041-1051.

(16) Kessenbrock, K., Plaks, V., and Werb, Z. (2010) Matrix metalloproteinases: regulators of the tumor microenvironment. Cell 141, $52-67$.

(17) Johnson, J. A., Lu, Y. Y., Van Deventer, J. A., and Tirrell, A. (2010) Residue-specific incorporation of non-canonical amino acids into proteins: recent developments and applications. Curr. Opin. Chem. Biol. 14, 774-780.

(18) Chalker, J. M., and Davis, B. G. (2010) Chemical mutagenesis: selective post-expression interconversion of protein amino acid residues. Curr. Opin. Chem. Biol. 14, 781-789.

(19) Rabuka, D. (2010) Chemoenzymatic methods for site-specific protein modification. Curr. Opin. Chem. Biol. 14, 790-796.

(20) Day, J. J., Marquez, B. V., Beck, H. E., Aweda, T. A., Gawande, P. D., and Meares, C. F. (2010) Chemically modified antibodies as diagnostic imaging agents. Curr. Opin. Chem. Biol. 14, 803-809. 